\title{
EFECTO DE UNA FITASA EN LA DIGESTIBILIDAD Y ACTIVIDAD DE TRIPSINA Y QUIMIOTRIPOSINA EN CERDOSDESTETADOS
}

\author{
EFFECT OF A PHTYASE FROM ASPERGILLUS NIGERON NUTRIENT DIGESTIBILITY \\ AND ACTIVITY OF TRYPSIN AND CHYMOTRYPSIN IN WEANLING PIGS
}

\begin{abstract}
Romero-Núñez, C. ${ }^{1}$, M. Salas-Ramírez ${ }^{1 *}$, A.C. García-Contreras ${ }^{1}$, G. Mendoza-Martínez¹, F.X. Plata-Pérez¹, M. Cervantes-Ramírez², T. Viana-Castrillón² y A. Morales-Trejo²
\end{abstract}

${ }^{1}$ Maestría en Ciencias Agropecuarias. Universidad Autónoma Metropolitana. Xochimilco. C.P. 04960, Distrito Federal. México. *Correspondencia: fiebrepc@senasica.sagarpa.gob.mx ${ }^{2}$ Universidad Autónoma de Baja California. Mexicali, B.C. México.

\section{PALABRAS CLAVES ADICIONALES \\ Lechones. Enzimas. Digestión. Páncreas.}

\section{RESUMEN}

Se realizaron dos experimentos para evaluar el efecto de la adición de fitasa fungal (Aspergillus niger) en la actividad de tripsina y quimotripsina y digestibilidad de nutrientes en cerdos cruzados (Yorkshire $\mathrm{x}$ Landrace, 11,5 kg peso vivo). En ambos experimentos se empleó una dieta base formulada con sorgo y pasta de soya; los tratamientos $(T)$ fueron los siguientes: $T 1$, dieta base, y T2, base adicionada con 500 unidades de actividad fitásica (FTU) por kg de alimento. En el exp. 1 se utilizaron seis cerdos canulados en conducto pancreático, los cuales fueron adaptados a las dietas durante $5 \mathrm{~d}$, previo a la colecta de muestras. La colección de jugo pancreático se hizo a intervalos de $15 \mathrm{~min}$, de 08:00 a 20:00 h, durante $5 \mathrm{~d}$. El alimento se ofreció dos veces al día (08:00 y 20:00 h). En el exp. 2 se utilizaron 20 cerdos alimentados con dietas similares del exp. 1 para estimar la digestibilidad total aparente de MS, MO, FDN, P, Ca, Mg, Zn y Fe. La fitasa no tuvo efectos en el peso y la longitud del páncreas, ni tampoco en la actividad de la tripsina y de la quimotripsina. La adición de fitasa incrementó la digestibilidad de la proteína $(p<0,10)$ de 78,81 a $81,60 \%$ y la de la FDN $(p<0,11)$ de 81,87 a $85,54 \%$. No se observaron diferencias en la digestibilidad del $\mathrm{Ca}, \mathrm{P}$ y $\mathrm{Fe}$. La digestibilidad de Mg y Zn tampoco se afectó por la adición de fitasa. En conclusión, estos resultados indican que la adición de fitasa fungal a dietas para cerdos no afecta la actividad de tripsina y quimotripsina ni la digestibilidad en tubo digestivo total de minerales en cerdos.

Recibido: 20-9-07. Aceptado: 21-2-08.
AdDitional KEYWORdS

Piglets. Enzymes. Digestion. Pancreas.

\section{SUMMARY}

Two experiments were conducted to evaluate the effects of addition of fungal phytase (Aspergillus niger) in activity of trypsin and chymotripsin and nutrient digestibility in crossed pigs (Yorkshire $x$ Landrace, $11.46 \mathrm{~kg} \mathrm{BW}$ ). In both experiments it was used a basal diet elaborated with sorghum and soybean meal, the treatments (T) were the following: T1, basal diet, and T2, basal plus 500 units of phytase activity (FTU) per $\mathrm{kg}$ In the exp. 1 six cannulated pigs in the pancreatic duct were used, which were adapted to the diets during 5 days before sample collection. The collection of pancreatic juice was done at $15 \mathrm{~min}$ interval from 08:00 to 20:00. The feed was offered twice per day. In the exp. 2, twenty pigs were fed with similar diets than exp. 1. The phytase did not show effects in the weight and the length of the pancreas, or either in the activity of the trypsin and chymotripsin. The addition of phytase increased the digestibility of protein $(p<0.10)$ from 78.81 to $81.60 \%$ and NDF $(p<0.11$ ) from 81.87 to $85.54 \%$. No differences were observed in digestibility of $\mathrm{Ca}, \mathrm{P}$ and $\mathrm{Fe}$. The digestibility of $\mathrm{Mg}$ and $\mathrm{Zn}$ were also not affected by phytase. In conclusion, these results indicate that the addition of fungal phytase to pig diets did not affect trypsin and chymotrypsin activity neither total mineral digestibility in gastrointestinal tract.

\section{INTRODUCCIÓN}

Los cereales (maíz, sorgo y trigo) y la Arch. Zootec. 58 (223): 363-369. 2009. 
pasta de soya, principales ingredientes de las dietas para cerdos, contienen niveles elevados de fitatos (Maga, 1982). La forma mas común de los fitatos es un éster de ácido fosfórico e inositol denominado ácido fítico o ácido hexafosfórico de mio-inositol (Reddy et al., 1982). Además del fósforo, los fitatos interactúan con otros minerales como el calcio, zinc, fierro y magnesio, y proteínas formando complejos fitato-minerales-proteínas. El resultado de la interacción es una reducción importante en la digestibilidad del fósforo, principalmente, seguida de los demás minerales y proteínas. La eficacia de la fitasa microbiana, enzima exógena que se adiciona de manera regular a las dietas para cerdos para liberar el fósforo de los fitatos, se ha documentado ampliamente (Cromwell, 1996).

Adicionalmente, se tienen evidencias in vitro de que los fitatos afectan negativamente la actividad de las enzimas proteolíticas pancreáticas. Singh y Krikorian (1982) encontraron in vitro una reducción sustancial en la actividad de tripsina debido a la presencia de fitatos. Este efecto lo atribuyeron a la formación de complejos fitato-enzima y a la formación de quelatos entre fitatos y los minerales requeridos para la activación de la enzima, reduciendo la actividad de la tripsina hasta $46 \%$.

En un estudio realizado con cerdas, $\mathrm{Mroz}$ et al. (1991) mostraron que la adición de fitasa aumentó la digestibilidad a nivel ileal de los compuestos nitrogenados en un 3\% y la actividad de tripsina en $10,9 \%$. Es posible que los efectos benéficos de la fitasa en la digestibilidad de la proteína se detecten sólo a nivel intestinal, en un experimento realizado por Radcliffe et al. (2006) en cerdas adultas con cánula ileocecal, con niveles de fitasa (0, 250 y 500 unidades), hubo respuesta lineal en la digestibilidad de la proteína $(\mathrm{p}<0,07)$ y aminoácidos $(\mathrm{p}<0,10)$, indicando que a nivel duodenal la disponibilidad de nutrientes es mayor, y que el efecto en heces puede no detectarse.

Las fitasas exógenas podrían tener dos efectos benéficos en la secreción y actividad de las enzimas pancreáticas, uno directo y otro indirecto En forma directa las fitasas podrían reducir la formación de los quelatos entre fitatos y tripsina (Singh y Krikorian, 1982) lo cual podría explicar la mayor actividad de la tripsina observada por Mroz et al. (1994). Un segundo mecanismo de acción indirecto, sería como consecuencia de una mayor digestibilidad de proteína y su consecuente absorción de aminoácidos en el intestino, lo cual podría estimular la colecistoquinina y la secreción pancreática (Jacob et al., 2000). Considerando lo expuesto, se realizó este estudio para evaluar el efecto de la adición de fitasa fungal elaboradas con sorgo-pasta de soya sobre la actividad de proteasas pancreáticas, digestibilidad y crecimiento de cerdos.

\section{MATERIAL Y MÉTODOS}

Se utilizaron 26 cerdos híbridos Yorkshire $\mathrm{x}$ Landrace, en etapa de crecimiento $(11,5 \mathrm{~kg}$ peso vivo). Seis cerdos se sometieron a un procedimiento de cirugía de canulación de conducto pancreático de acuerdo al procedimiento descrito por Romero (2006) y los 20 restantes se usaron en un ensayo de digestibilidad y comportamiento productivo. Los cerdos canulados se alojaron en jaulas individuales, a $28 \pm 2{ }^{\circ} \mathrm{C}$ y $45 \pm 2 \%$ humedad relativa. Los 20 cerdos sin canular se alojaron en jaulas con piso elevado y se asignaron aleatoriamente a dos tratamientos: a) fitasa fungal en el alimento y b) grupo testigo sin fitasa; cada tratamiento estuvo formado por 10 repeticiones.

Los cerdos no canulados fueron alimentados con dietas sólidas para crecimiento (tabla I), durante siete días de adaptación. Posteriormente se les ofreció alimento adicionado 500 unidades fitasa (FTU) de Aspergillus niger (Natuphos ${ }^{\circledR}$ BASF) por $\mathrm{kg}$ de dieta, durante 7 días y agua ad libitum. La dieta fue formulada de acuerdo con NRC (1998). A los cerdos canulados se les dio un periodo de adaptación a la dieta (tabla I) y 


\section{FITASASEN CERDOS DESTETADOS, DIGESTIBILIDAD Y ENZIMAS PANCREÁTICAS}

la cánula de cinco días, posteriormente a la mitad de los cerdos (3) se les ofreció alimento adicionado con $2500 \mathrm{UF}$ por $\mathrm{kg}$ de dieta, durante cinco días. Estos cerdos se alimentaron ad libitum y se les registró el consumo debido a que el tiempo se dedicó al muestreo de jugo pancreático. La colección de jugo pancreático se hizo a intervalos de $15 \mathrm{~min}$, de 08: 00 a 20: $00 \mathrm{~h}$, durante 5 días.

Se hizo una muestra compuesta de heces por cada cerdo, para determinar los nutrientes y las cenizas insolubles en ácido como marcador interno. Se determinó la materia seca, materia orgánica (AOAC, 1990), fibra detergente neutro (Van Soest et al., 1991), fósforo total por la técnica de Harris y Popat (1954), y otros minerales (calcio, magnesio, zinc y hierro), por espectrofotometría de absorción atómica (Slavin, 1968).

Se analizaron las cenizas ácido insolubles (Keulen y Young, 1977), para calcular la digestibilidad in vivo con las siguientes ecuaciones (Osuji et al., 1993):

Digestibilidad nutriente $=100-\left[\left(100^{*} \mathrm{CIA} \mathrm{dx}\right.\right.$

Tabla I. Composición porcentual de las raciones usadas para los cerdos canulados y para el ensayo de digestibilidad. (Percent composition of diets used for cannulated pigs and for digestibility assay).

\begin{tabular}{lcc}
\hline Ingrediente & $\begin{array}{c}\text { Cerdos } \\
\text { canulados }\end{array}$ & $\begin{array}{c}\text { Ensayo de } \\
\text { digestibilidad }\end{array}$ \\
\hline Pasta de soya & 20,566 & 27,00 \\
Sorgo grano & 75,553 & 69,00 \\
Aceite de soya & 1,042 & 1,00 \\
Premezcla vitamínica & 0,250 & 0,27 \\
Premezcla mineral & 0,150 & 0,19 \\
Sal & 0,350 & 0,12 \\
Saborizante & - & 0,10 \\
Carbonato de calcio & 1,112 & 1,00 \\
Fosofato monodicálcico & 0,684 & - \\
Ortofosfato & - & 0,98 \\
L-Lisina HCl & 0,259 & 0,14 \\
DL-Metionina & 0,014 & 0,17 \\
L-Treonina & 0,020 & 0,02 \\
L-Triptofano & - & 0,01 \\
\hline
\end{tabular}

nutriente $h) /(C I A h+$ nutriente $d)]$

donde:

$\mathrm{CIA} d=$ cenizas insolubles en ácido en la dieta $(\%)$ $\mathrm{CIA} h=$ cenizas insolubles en ácido en heces $(\%)$. Nutriente $\mathrm{h}=$ nutriente en heces $(\%)$.

Nutriente $\mathrm{d}=$ nutriente en dieta $(\%)$.

La actividad enzimática en jugo pancreático se analizó en jugo pancreático la proteína soluble por el método de Bradford (1976) usando seroalbúmina bovina como estándar. Las enzimas fueron activadas con enteroquinasa porcina (Sigma). La actividad de la tripsina se determinó por el método de Erlanger et al. (1961) usando benzoil-Larginina-etil-ester como substrato. Una unidad de actividad de tripsina fue igual a la hidrólisis de $1 \mu \mathrm{mol}$ de substrato por minuto por $\mathrm{mg}$ de proteína. La actividad de la quimotripsina se determinó con la hidrólisis de N-acetil-L-tirosina-etil-ester (Hibbard et al., 1992). Una unidad de actividad de quimotripsina fue igual a la hidrólisis de 1 $\mu$ mol de substrato por minuto por $\mathrm{mg}$ de proteína.

\section{ANÁLISISESTADÍSTICOS}

Se realizó el análisis de varianza de las variables respuesta con un modelo estadístico con un solo criterio de clasificación (nivel de fitasa): El modelo estadístico usado fue:

$$
Y_{\mathrm{ijkl}}=\mu+\mathrm{P}_{\mathrm{i}}+\varepsilon_{\mathrm{ijkl}}
$$

donde:

$\mathrm{Y}_{\mathrm{ijklm}}=$ variable respuesta.

$\mu=$ media general.

$\mathrm{P}_{\mathrm{i}}=$ efecto del nivel de fitasa.

$\varepsilon_{\mathrm{ijk}}=$ error aleatorio.

Los resultados de ganancia de peso se analizaron usando el peso inicial como covariable (Steel y Torrie, 1996). Los resultados de actividad enzimática (tripsina y quimotripsina), se analizaron con el procedimiento de mediciones repetidas del SAS (1999). 


\section{RESULTADOSYDISCUSION}

De los nutrientes analizados (tabla II), la adición de la fitasa fungal de Aspergillus niger sólo incrementó la digestibilidad de la proteína $(\mathrm{p}<0,10)$ en 2,8 unidades porcentuales y la de la fracción de la FDN en 3,6 unidades $(\mathrm{p}<0,11)$. Pero, no se observaron diferencias $(p=0,10)$ en las digestibilidades de los otros componentes de la dieta ni de los minerales. El fitato se considera como agente antinutricional, debido a que forma complejos (quelatos) con gran variedad de minerales esenciales como $\mathrm{Ca}, \mathrm{Zn}, \mathrm{Mg}$ y Fe, puede reaccionar con proteínas reduciendo su disponibilidad (Reddy et al., 1982), lo cual explica que la adición de fitasas mejore la disponibilidad de la proteína.

Los resultados en este experimento, son similares a los reportados en la literatura. En evaluaciones con cerdos en crecimiento y finalización, Harper et al. (1997) al incrementar la dosis de la fitasa Natuphos ${ }^{\circledR}$ de 250 a $500 \mathrm{U}$ por kg de alimento; tampoco no encontraron respuesta en la digestibilidad de calcio en dietas con baja concentración de fósforo, a pesar de haberse estimado que la digestibilidad del $\mathrm{P}$ se podría incrementar en un 30\%; tampoco encontraron efecto en la digestibilidad de la materia seca. Por su parte, Olukosi et al. (2007) evaluaron dosis crecientes de fitasas $(0,500$ y 1000 unidades de fitasa) en cerdos destetados y observaron una respuesta lineal en la digestibilidad del fósforo $(47,49$ y $56 \%$ ) y del calcio $(53,60$ y $68 \%$ ), sin encontrar efectos en la materia seca, proteína y energía. Adeola et al. (1995) observaron que con 1500 unidades de fitasa en el alimento, se incrementaba la disponibilidad aparente de $\mathrm{Ca}, \mathrm{P}, \mathrm{Zn}$ y $\mathrm{Cu}$, sin embargo, no encontraron efectos en la digestibilidad de $\mathrm{Mg}$ y $\mathrm{Mn}$.

En un experimento con cerdos suplementados con Natuphos ${ }^{\circledR}(0,250$ y 500 Unidades por $\mathrm{kg}$ de dieta) realizado por Radcliffe et al. (2006) en cerdas adultas con cánula ileocecal, detectaron efectos en la digestibilidad ileal de la fitasa en la digesti-
Tabla II. Efecto de una fitasa fungal (500 unidades/kg alimento) en la digestibilidad aparente de nutrientes y en el comportamiento de lechones destetados. (Effect of fungal phytase (500 units/kg feed) on apparent digestibility of nutrientes and perfomance of weaning pigs).

\begin{tabular}{lrrrr}
\hline Item & Testigo & Fitasa & C.V.(\%) & $\mathrm{P}^{1}$ \\
\hline Digestibilidad: & & & & \\
Materia seca (\%) & 87,58 & 88,55 & 2,16 & 0,27 \\
Proteína (\%) & 78,81 & 81,60 & 4,44 & 0,10 \\
FDN (\%) & 81,87 & 85,54 & 5,77 & 0,11 \\
Ca (\%) & 71,57 & 77,56 & 15,79 & 0,28 \\
P (\%) & 51,73 & 53,98 & 19,08 & 0,63 \\
Mg (\%) & 94,32 & 93,47 & 4,31 & 0,65 \\
Zn (\%) & 92,47 & 92,79 & 3,91 & 0,92 \\
Fe (\%) & 86,42 & 88,62 & 5,66 & 0,36 \\
Peso inicial & 11,24 & 11,68 & 15,35 & 0,58 \\
Peso final $^{2}$ & 13,88 & 14,75 & 11,90 & 0,26 \\
Ganancia diaria $^{2}$ & $0,187^{\mathrm{a}}$ & $0,219^{\mathrm{b}}$ & 13,67 & 0,02 \\
\hline
\end{tabular}

${ }^{1}$ Probabilidad de error tipo I; ${ }^{2} \mathrm{~kg}$.

abMedias con distinta literal son diferentes $(p<0,05)$.

bilidad del $\mathrm{P}$ (lineal, $\mathrm{p}<0,01), \mathrm{Ca}(\mathrm{p}<0,004)$, MS ( $p<0,11)$, proteína $(\mathrm{p}<0,07)$ y aminoácidos $(p<0,10)$. Pero cuando midieron la digestibilidad en las heces, no detectaron efectos en la MS ni en la energía, pero sí tuvieron efecto lineal en las de $\mathrm{P}(\mathrm{p}<0,01)$ y $\mathrm{Ca}(\mathrm{p}<0,12)$. Este experimento nos indica que es posible que las fitasas tengan efecto a nivel intestinal, pero que no siempre se pueda detectar el efecto al medir la digestibilidad en heces, lo cual explicaría muchas de las faltas de consistencia de efectos reportadas en la literatura.

Algunos estudios donde se han evaluado dosis mayores a las 500 unidades, se han logrado detectar efectos en la digestibilidad aparente de la MS y de otros nutrientes. La mayoría de los estudios muestran efectos más consistentes sobre el fósforo, y se puede concluir que el uso de fitasas permite reducir la excreción de $\mathrm{P}$ y la contaminación de este mineral hasta en un 30 a 40\% (Harper etal., 1997).

Se ha reportado que el fitato puede for- 


\section{FITASASEN CERDOS DESTETADOS, DIGESTIBILIDAD Y ENZIMAS PANCREÁTICAS}

mar sales insolubles con $\mathrm{Ca}, \mathrm{Fe}, \mathrm{Zn}, \mathrm{Mn}$ y $\mathrm{Cu}$ disminuyendo la disponibilidad de esos minerales. Adeola et al. (1995) indicaron que la digestibilidad del Zn se mejoró al adicionar fitasa a la dieta de cerdos destetados. Sin embargo, en este estudio no se ha encontrado efecto de la fitasa en la digestibilidad de Mg, Zn y Fe (tabla II). A pesar de que no siempre se detecten efectos de la fitasa, la degradación del ácido fítico reduciría la formación de complejos solubles y quelatos insolubles con diversos minerales como Zn, Cu, Co, Mn, Ca y Fe (Maenz et al., 1999) incrementando la disponibilidad para su absorción.

Las investigaciones con fitasas se han enfocado a micro minerales y aminoácidos, y solamente en algunos estudios se han combinado con enzimas fibrolíticas, pero no se han medido los efectos en la digestibilidad en la FDN. En un experimento con aves, la combinación de fitasas con xilanasas mejoró la energía metabolizable del alimento (Ravindran et al., 1999), mientras que en otros estudios no ha obtenido respuesta (Juanpere et al., 2005), por lo que no existe evidencia directa de que las fitasas puedan mejorar la digestión de la FDN. No obstante, es importante tener presente que el ácido fítico es un componente común de las plantas y es almacenado en un complejo grande con sales de $\mathrm{Mg}$ y $\mathrm{K}$ junto con proteínas dentro de membranas simples en partículas de granos y semillas (López et al., 2000). Esto explicaría por qué en este experimento la adición de las fitasas tendió a mejorar $(\mathrm{p}=$ 0,11 ) la digestibilidad de la FDN en cerdos.

En este estudio, la adición de fitasa mejoró $(\mathrm{p}<0,05)$ la ganancia de peso (tabla II) lo cual podría asociarse a la mayor disponibilidad de proteína de la dieta. Gómez et al. (2002) mostraron que el crecimiento de cerdos se puede mejorar al tener un mayor suministro de aminoácidos digestibles de lisina, treonina, triptófano y metionina, que es lo que pudo haber ocurrido como efecto indirecto al haber suministrado la fitasa. Los mayores crecimientos al adicionar fita- sa en cerdos son el resultado de un mayor consumo de nutrientes, en los cuales la proteína tiene un impacto determinante y que se reporta en varios experimentos.

Otro nutriente que ha sido responsabilizado de causar mayor crecimiento es el fósforo. Harper et al. (1997) observaron mayores ganancias de peso con la fitasa en dietas con baja concentración de fósforo en cerdos en crecimiento y finalización asociadas a mayor consumo de alimento y a una mayor digestibilidad de fósforo.

La adición de fitasa no tuvo efectos $(\mathrm{p}<0,05)$ en el peso y la longitud del páncreas, ni tampoco en la actividad de la tripsina y de la quimotripsina en los 5 días de colección de jugo pancreático (tabla III), por lo que el efecto de evitar la formación de quelatos fue prácticamente nulo. Solamente se observó una tendencia a mayor actividad de la tripsina $(p=0,11)$ en el primer día de

Tabla III. Efecto de una fitasa fungal (500 unidades/kg alimento) en características del páncreas y actividad de tripsina y quimotripsina. (Effect of fungal phytase (500 units/kg feed) on pancreas characteristics and activity of trypsin and quimotrypsin).

\begin{tabular}{|c|c|c|c|c|}
\hline & Testigo & Fitasa & CV (\%) & $\mathrm{P}^{1}$ \\
\hline \multicolumn{5}{|l|}{ Páncreas } \\
\hline peso $(g)$ & 32,62 & 33,34 & 10,24 & 0,63 \\
\hline longitud (mm) & 14,16 & 14,40 & 3,13 & 0,25 \\
\hline \multicolumn{5}{|c|}{ Tripsina (Unidades/mg proteína) } \\
\hline Día 1 & 3,785 & 4,184 & 6,12 & 0,11 \\
\hline Día 2 & 3,951 & 4,276 & 7,44 & 0,26 \\
\hline Día 3 & 3,835 & 3,673 & 10,14 & 0,63 \\
\hline Día 4 & 3,731 & 3,661 & 27,35 & 0,93 \\
\hline Día 5 & 3,752 & 3,893 & 11,64 & 0,71 \\
\hline \multicolumn{5}{|c|}{ Quimotripsina (Unidades/mg proteína) } \\
\hline Día 1 & 2,191 & 3,731 & 41,23 & 0,19 \\
\hline Día 2 & 2,391 & 3,222 & 39,73 & 0,41 \\
\hline Día 3 & 2,789 & 3,811 & 23,14 & 0,17 \\
\hline Día 4 & 2,510 & 2,421 & 60,01 & 0,94 \\
\hline Día 5 & 3,076 & 2,680 & 34,03 & 0,62 \\
\hline
\end{tabular}

${ }^{1}$ Probabilidad de error tipo I. 
suministro de la fitasa. Mroz et al. (1991) reportaron una mayor actividad de la tripsina al adicionar fitasa. Si bien se había considerado que la posible respuesta en páncreas sería mediada por efecto indirecto de los aminoácidos (Niederau et al., 1986), y esto estaría reflejado en la digestibilidad de la proteína, no se obtuvo evidencia para probar esta hipótesis, pues la mayor digestibilidad de la proteína no está asociada a mayor actividad de las proteasas. Los resultados del grupo testigo no mostraron evidencia de que el ácido fítico haya formado complejos con la proteína, inhibiendo la tripsina y la pepsina como se ha reportado (Mroz etal., 1994).

\section{BIBLIOGRAFÍA}

Adeola, O., B.V. Lawrence, A.L. Sutton and T.B. Cline. 1995. Phytase-induced changes in mineral utilization in zinc supplemented diets for pigs. J. Anim. Sci., 73: 3384-3391.

AOAC. 1990. Official Methods of Analysis of the Association of Official Analytical Chemists. Vol 1. $15^{\text {th }}$ Ed. Assoc. Offic. Anal. Chem. Washington, D.C. p. 69-88.

Bradford, M.M. 1976. A rapid and sensitive method for the quantitation of microgram quantities of protein utilizing the principle of protein-dye binding. Anal. Biochem., 72: 248-252.

Cromwell, G.L. 1996. The effects of phytase on phosphosrus utilization in swine. BASF Technical Symposium. Des Moines. Lowa. $p$. 70-93.

Erlanger, B.F., N. Kokowski and W. Cohen. 1961. The preparation and propierties of two new chormogenic substrates of trypsin. Arch. Biochem. Biophys., 95: 271-278.

Gómez, R.S., A.J. Lewis, P.S. Miller and H.Y. Chen. 2002. Growth performance, diet apparent digestibility, and plasma metabolite concentrations of barrows fed corn-soybean meal diets or low-protein, aminoacid-supplemented diets at different feeding levels. J. Anim. Sci. 80: 644-653.

Harper, A.F., E.T. Kornegay and T.C. Schell. 1997. Phytase supplementation of low phosphorous growing-finishing pig diets improves perfor-

\section{CONCLUSIONES}

El uso de fitasa fungal en dietas para cerdos destetados incrementó la digestibilidad de proteína y favoreció una mayor ganancia de peso, sin modificar la digestibilidad de otros nutrientes de la dieta, ni la actividad de tripsina y quimotripsina.

\section{AGRADECIMIENTOS}

Se agradece la participación de los estudiantes de Medicina Veterinaria y Zootecnia de la Universidad Autónoma Metropolitana, Alejandro Martínez Jiménez, Efraín Hernández Ramírez, Mariana Dafne Carrillo del Valle y Fernando León Hernández.

mance, phosphorous digestibility, and bone mineralization and reduces phosphorous excretion. J. Anim. Sci., 75: 3174-3186.

Harris, W.D. and P. Popat. 1954. Determination of the phosphorus content of lipids. Amer. Oil Chem. Soc. J., 31: 124.

Hibbard, B., J.P. Peters, R.Y.W. Shen and S.T. Chester. 1992. Effect of recombinant porcine somatotropin and dietary protein on pancreatic digestive enzymes in the pig. J. Anim. Sci., 70: 2188-2194.

Jacob S., Mosenthin, R. Zabielski, R. Rippe, C. Winzell, M.S. Gacsalyi, U. Laubitz, D. Grzesiuk and S.G. Pierzynowski. 2000. Fats infused intraduodenally affect the postprandial secretion of the exocrine pancreas and the plasma concentration of cholecystokinin but not of peptide $Y Y$ in growing pigs. J. Nutr., 130: 24502455.

Juanpere, J., V.A.M. Pérez, E. Angulo and J. Brufau. 2005. Assesment of potential interaction bewteen phytase and glycosidase enzyme supplementation on nutrient digestibility in broilers. Poultry Sci., 84: 571-580.

Keulen, J.V. and B.A. Young. 1977. Evaluation of acid-insoluble ash as a natural marker in ruminant digestibility studies. J. Anim. Sci., 44: 282-287.

López, H.W., F. Vallery, M.A. Levrat, Ch. Coudray, Ch. Demigne and Ch. Rémésy. 2000. Dietary phytic acid and wheat bran enhance mucosal

Archivos de zootecnia vol. 58, núm. 223, p. 368. 


\section{FITASAS EN CERDOS DESTETADOS, DIGESTIBILIDAD Y ENZIMAS PANCREÁTICAS}

phytase activity in rat small intestine. J. Nutr. 130: 2020-2025.

Maenz, D.D., C.M. Engele-Schann, R.W. Newkirk and H.L. Classen. 1999. The effect of minerals and mineral chelators on the formation of phytateresistant and phytase-susceptible forms of phytic acid in solution and in a slurry of canola meal. Anim. Feed Sci. Tech., 77: 177-192.

Maga, J.A. 1982. Phytate: its chemistry, occurrence, food interactions, nutritional significance, and methods of analysis. J. Agric. Food Chem., 30: 1-9.

Mroz, Z., A.W. Jongbloed, A. Kemme and N.P Lenis. 1991. Ileal and overall digestibility of nitrogen and amino acids in a diet for pigs as influenced by Aspergillus niger phytase and feeding frequency levels. Proc. $6^{\text {th }}$ Int. Sym. Protein Metab. Nutr. Herning Denmark. 225 p.

Mroz, Z., A.W. Jongbloed and A.P. Kemme. 1994. Apparent digestibility and retention of nutrient bound to phytate complexes as influenced by microbial phytase and feeding regimen in pigs. J. Anim. Sci., 72: 126-132.

Niederau, C., J.H. Grendell and S.S. Rothman 1986. Digestive end products release pancreatic enzymes from particular cellular pools, particularly zymogen granules. Biochim. Bophys. Acta, 881: 218-291.

NRC. 1998. Nutrient Requirements of Swine $\left(9^{\text {th }}\right.$ Ed.). National Academy Press. Washington, DC Olukosi, O.A., J.S. Sands and O. Adeola. 2007. Supplementation of carbohydrases or phytase individually or in combination to diets for weanling or growing finishing-pigs. J. Anim. Sci., 85: 1702-1711.
Osuji, P.A., I. Nsahlai and H. Kahlili. 1993. Feed evaluation. ILCA Manual. Internacional Livestock Center for Africa. $40 \mathrm{p}$.

Radcliffe, J.S., R.S. Pleasant and E.T. Kornegay, 2006. Estimating equivalency values of microbial phytase for amino acids in growing and finishing pigs fitted with steered ileo-cecal calve cannulas. J. Anim. Sci., 84: 119-1129.

Ravindran, V., P.H Selle and W.L Bryden. 1999. Effects of phtyase supplementation, individually and in combination, with glycanase, on the nutritive value of wheat and barley. Poultry Sci., 78: 1588-1595.

Reddy, N.R., S.K. Sathé and D.K. Salunkhe. 1982. Phytates in legumes and cereals. Adv. Food Res., 28: 1-92.

Romero, N.C. 2006. Implantación quirúrgica de una cánula en el conducto pancreático en cerdos. Tesis de Maestría en Ciencias Agropecuarias. Universidad Autónoma Metropolitana, Unidad Xochimilco. $55 \mathrm{p}$.

SAS. 1999. SAS User's Guide Statistics, version 8.1. SAS Inst. Inc. Cary. North Carolina.

Singh, M. and A.D. Krikorian. 1982. Inhibition of trypsin activity by phytate. J. Agric. Food Chem., 30: 799-800.

Slavin, W. 1968. Atomic absortion spectroscopy. John Wiley and Sons. N.Y.

Steel, R.G.D. y J.H. Torrie. 1996. Bioestadística: Principios y procedimientos. $2^{a}$ Edición. McGrawHill. $622 \mathrm{p}$.

Van Soest, P.J., J.B. Robertson and B.A. Lewis. 1991. Symposium: Carbohydrate methodology, metabolism, and nutritional implications in dairy cattle. J. Dairy Sci., 74: 3583-3697. 